\title{
MEDICINA BASADA EN LA EVIDENCIA: BÚSQUEDA EN BASES DE DATOS EN INTERNET Y TÉRMINOS MeSH
}

VIDENCE BASED MEDICINE: SEARCH ON INTERNET DATABASES AND MESH TERMINOLOGY

MEDICINA COM BASE NA EVIDENCIA: PESQUISA EM BASE DE DADOS NA INTERNET E TERMOS MeSH

\author{
MARÍA CAROLINA DUARTE MARTÍNEZ \\ Universidad Católica de Santiago de Guayaquil, Guayaquil, Ecuador
}

\begin{abstract}
Resumen
Las bases de datos en internet son el espacio donde se encuentra la información médica más actualizada en forma de artículos, por este motivo es importante adquirir el máximo beneficio de las mismas. Sin embargo, muchas veces no se consigue todo su potencial por el desconocimiento de las estrategias de búsqueda por parte de la comunidad médica. Los motores de búsqueda científica se encuentran al alcance de todos en la red y nos permiten el acceso a un sin número de información, mucha de esta gratuita; pero al mismo tiempo, al ser tan abundante, puede resultar en ocasiones extenuante para el investigador si no delimita su búsqueda. Es por esto que se recomienda la utilización de términos estandarizados, como los DeSC (Descriptores en Ciencia de la Salud) o MeSH (Medical Subject Headings), para ser más eficientes pudiendo ahorrarse tiempo y energía. En este artículo se presenta las bases de datos más conocidas y se detallan consejos de cómo encontrar referencias bibliográficas de alto nivel de evidencia.
\end{abstract}

PALABRAS CLAVE: resumen e indización como asunto, bibliografía, internet, Medical Subject Headings, descriptores.

Abstract

Internet databases are places where the most up-to-date medical information is found in the form of articles. For this reason it is important to achieve maximum benefit out of them. However, many times their full potential is not reached due to ignorance of search strategies among the medical community. Scientific search engines are available to everyone in the network and allow access to a great deal of information, and a lot of it, for free; but at the same time, abundance can sometimes be exhausting for researchers if they do not delimit their search. This is why it is recommended to use standardized terms, such as DeSC (Descriptors in Health Science) or MeSH (Medical Subject Headings), to be more efficient and save time and energy. In this article, the best-known databases are presented along with tips on how to find highly evidence-based literature.

KEYWORDS: abstracting and indexing as topic, bibliography, internet, medical subject headings, descriptors.

Resumo

As bases de dados na internet são o espaço onde é encontrada a informação medica mais atualizada em forma de artículos, portanto é importante adquirir o máximo beneficio delas. Porém, muitas vezes não se consegue todo o seu potencial por causa do desconhecimento das estratégias de pesquisas pela comunidade medica. Os motores de pesquisa cientifica estão disponíveis para todos na internet, e nos permitem 0 acesso a uma informação infinita, muita desta gratuita; mas ao mesmo tempo, ao ser tão abundante, pode resultar extenuante para o investigador, se este não delimitar a sua pesquisa. É por isso que É recomendado o uso de termos estandardizados, como os DeSC (Descritores em Ciência e Saúde) o MeSH (Medical Subject Headings), para ser mais eficientes, sendo possível economizar tempo e energia. Neste articulo, apresentamos as bases de dados mais conhecidas e se compartilham dicas de como encontrar referencias bibliográficas de alto nível de evidencia.

PALABRAS-CHAVE: resumos e indexação como assucto, bibliografia, internet, medical subject headings, descritores. 
INTRODUCCIÓN

A lo largo de los años la búsqueda en bases de datos ha ido poniendo a nuestra disposición todo tipo de información. Sin embargo, muchos profesionales en la salud no han desarrollado las habilidades en el manejo de los motores de búsqueda. ${ }^{1}$ En la actualidad la cantidad de resultados de investigaciones es impresionante. La implementación y conocimiento expansivo del internet ha permitido el acceso en cualquier parte del mundo a todo tipo de información. ${ }^{2}$ Esto representa, en el ámbito de la salud, una inmensa extensión de conocimientos para actualización, aprendizaje e investigación médica. ${ }^{3}$ Es por este motivo que es necesario aprender a descartar y escoger información usando estrategias en búsqueda validada científicamente para la toma de decisiones médicas. ${ }^{3,4}$ Es decir, que todo trabajo publicado guarda una responsabilidad de índole legal. Los motores de búsqueda generales recogen información de calidad discutible, por lo que es preferible utilizar buscadores biomédicos. ${ }^{5}$ Además la búsqueda de artículos en internet permite encontrar una amplia variedad de artículos que podría confundir al investigador y producir el desgaste y cansancio a la persona y la desmotivación en seguir el proceso. ${ }^{1,6}$

Existen varias bases de datos en internet de fácil acceso, por lo que resulta conveniente conocer de ellas y como utilizarlas. ${ }^{7}$ Las principales, con sus respectivos enlaces, se muestran en la tabla 1.

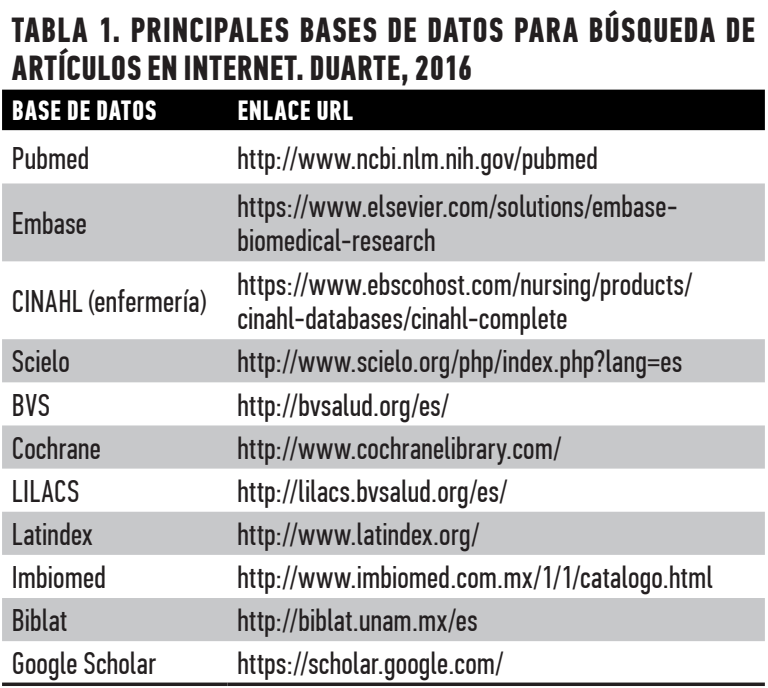

BASES DE DATOS EN INTERNET

La base de datos más reconocida y utilizada es Pubmed, la cual corresponde a MEDLINE, la biblioteca médica del Instituto Nacional de Salud (NIH) de los Estados Unidos. ${ }^{8,9}$ Esta base de datos tiene más de catorce millones de citaciones, de más de 4800 revistas, en varios campos de las ciencias de la salud, y en su mayoría en idioma inglés. Se actualiza todos los días entre martes y sábado, e incluye artículos a partir del año $1966 .{ }^{3} \mathrm{Su}$ acceso es gratuito y ofrece a los usuarios la opción de crear una cuenta para guardar sus búsquedas y facilitar la recolección de datos posterior a la navegación. ${ }^{3}$

El uso de Pubmed es relativamente sencillo y además ofrece opciones de búsqueda avanzada para mejorar la calidad del material encontrado. ${ }^{10,11}$ Al ingresar a la página inicial, bajo el buscador principal existe el vínculo "Advanced", que nos lleva al constructor de búsqueda de Pubmed. En esta nueva ventana, a nuestra disposición se ofrecen menús desplegables que permiten refinar la búsqueda, bien sea por el nombre de un autor, el título de una revista o una palabra clave en relación a la enfermedad de interés, entre otras. Además, permite la utilización de los conectores [AND], que se utilizan para enlazar dos términos de búsqueda; [OR] para mostrar opciones de búsqueda, y [NOT] para evitar artículos que contengan determinados términos. Se pueden colocar tantos términos y conectores como sean necesarios, estos aseguran la exactitud de la búsqueda. ${ }^{3,11,12}$ Por último, esta página permite el acceso al historial del investigador, asegurando que la información de las búsquedas realizadas no se pierda. Adicionalmente, al obtener los resultados, en el lado izquierdo de la pantalla se nos permite agregar nuevos parámetros para filtrar la búsqueda y enfocarla aún más, como el tipo de artículo, año de publicación, idioma, y demás. (Figura 1). Una base de datos muy conocida es Embase, alojada en el servidor de la editorial Elsevier. Esta tiene su origen en Europa, y en ella se concentran la mayoría de publicaciones de dicho continente. ${ }^{13} \mathrm{~A}$ pesar de los pocos trabajos existentes sobre su utilización, contiene cerca de nueve millones de citaciones por parte de casi 4600 revistas, y se añaden entre 6000 a 8000 citaciones semanalmente ${ }^{3,14}$. Una de las desventajas que presenta Embase es la necesidad de iniciar sesión con una cuenta personal para poder buscar artículos. Existen otras ramas de la salud como enfermería, cuya base de datos más reconocida es la Cumulative Index to Nursing and Allied Health (CINAHL), la cual posee un alto grado de superposición con los artículos alojados en MEDLINE referentes a este campo. Tiene más de 1.7 millones de citaciones, con más de 140 revistas indexadas. ${ }^{3,15}$ 


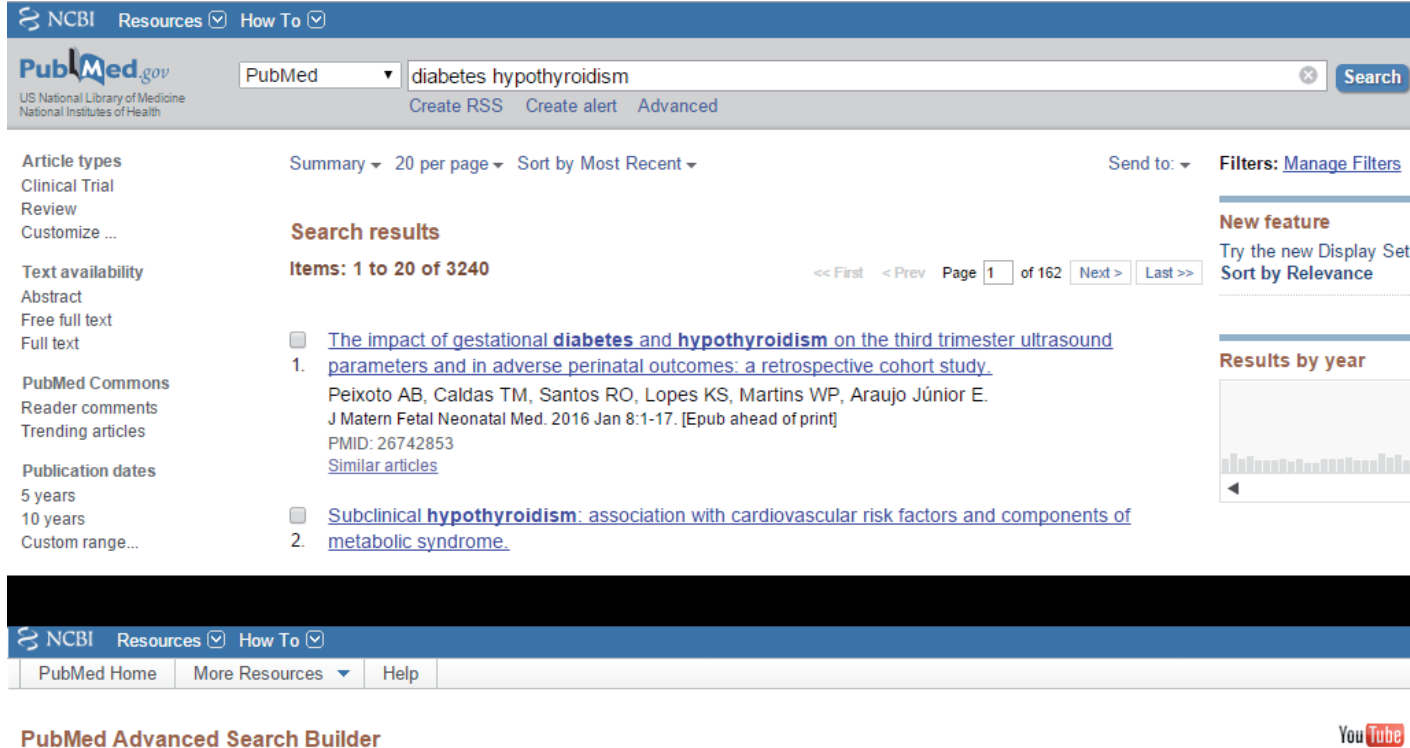

((diabetes mellitus) AND hypothyroidism) NOT type 2 diabetes

Edit

Builder

\begin{tabular}{|c|c|c|c|}
\hline & All Fields & diabetes mellitus & Show index lis \\
\hline AND $\boldsymbol{v}$ & All Fields & hypothyroidism & Show index lis \\
\hline NOT $\mathbf{v}$ & All Fields & type 2 diabetes & $\bullet \oplus$ Show index \\
\hline
\end{tabular}

NOT $\checkmark$ All Fields

- type 2 diabetes

Search

or Add to history

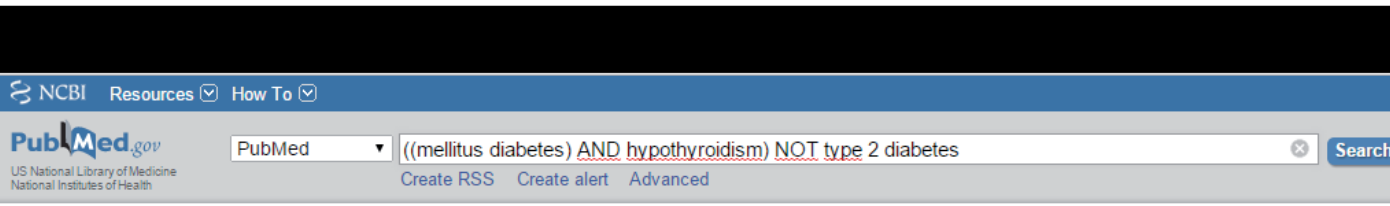

Summary 20 per page $\mathbf{v}$ Sort by Most Recent $\mathbf{v}$

Send to: Filters: Manage Filters

See 8 citations found by title matching your search

Association between subclinical hypothyroidism and diabetic nephropathy in patients with type 2 diabetes mellitus. Furukawa S et al. Endocr J. (2014)

Type 2 diabetes mellitus and hypothyroidism: the possible influence of metformin therapy. Distiller LA

al. Diabet Med. (2014)

IImpact of replacement therapy for subclinical hypothyroidism on cardiovascular risk markers in patients with type 2 diabetes mellitus]. Kasatkina SG et al. Ter Arkh. (2012)

New feature

Review

Text availability

Full text

PubMed Commons

Reader comments

Trending articles

Search results

Publication dates

Items: 1 to 20 of 1411

« First < Prev Page 1 of 71 Next $>$ Last $\gg$

5years

Custom range...

The impact of gestational diabetes and hypothyroidism on the third trimester ultrasound

1. parameters and in adverse perinatal outcomes: a retrospective cohort study.

Peixoto AB, Caldas TM, Santos RO, Lopes KS, Martins WP, Araujo Júnior

J Matern Fetal Neonatal Med. 2016 Jan 8:1-17. [Epub ahead of print]

Humans

Other Animals

Clear all

PMID: 26742853

Show additional filters

Prevalence of CHD-related metabolic comorbidity of diabetes mellitus in Northern Chinese adults:

2. the REACTION study

Gao N, Yuan Z, Tang X, Zhou X, Zhao M, Liu L, Ji J, Xue F, Ning G, Zhao J, Zhang H, Gao L.

J Diabetes Complications. 2015 Nov 24. pii: $S 1056-8727$ (15)00456-0. doi: 10.1016/j.jdiacomp. 2015.11.018. [Epu

ahead of print]

PMID: 2671893

Similar articles

Titles with your sean

Diabetes mellitus and Strange bedfellows or $n$ Mutations in GLIS3 are syndrome with neonatal

Multiple endocrinopathie deficiency, autoimmune

Find related data

Figura 1. Ejemplo de búsqueda avanzada en Pubmed. Al buscar simplemente "diabetes" e "hipotiroidismo", nos ofrece una larga lista de resultados no filtrados. Por otro lado, al utilizar el buscador avanzado podemos utilizar el término MeSH adecuado (diabetes mellitus), añadir conectores y pedir que nos rechace los resultados que traten de diabetes tipo 2 (no relacionada con hipotiroidismo), obteniendo así una lista de resultados con menos de la mitad obtenidos originalmente. Nótese del lado izquierdo en la última imagen, las opciones de filtro a los resultados que se mencionan en el texto, útiles para refinar aún más la búsqueda. 
Otras bases de datos utilizadas, especialmente a nivel de Latinoamérica, son las bibliotecas de Scielo, la cual tiene subdivisiones para varios países de la región, así como la Biblioteca Virtual en Salud (BVS), alojada en Brasil, que incluye a la gran mayoría de los artículos publicados en Sudamérica. Si el objetivo es buscar revisiones sistemáticas, con un mayor nivel de evidencia, es recomendable utilizar la base de datos de The Cochrane Collaboration, que ofrece la mayor cantidad de este tipo de artículos con una alta calidad. Otros repositorios en español incluyen LILACS (parte de BVS), Latindex, Imbiomed y Biblat. Además, hace unos años el buscador Google lanzó Google Scholar, que se enfoca en buscar solamente artículos científicos. 5,7

Todas las bases de datos ofrecen libre acceso a los resúmenes de cada artículo seleccionado, pero no todos estos trabajos tienen acceso gratuito al texto completo en sus revistas, hecho que puede dificultar el inicio de la revisión y redacción del manuscrito una vez que se han seleccionado los artículos tras la búsqueda. ${ }^{4}$ Algunas revistas permiten la visualización completa luego de cierto tiempo desde que fueron publicadas. Existe la ventaja del acceso institucional a las revistas, este generalmente es otorgado a campus universitarios y hospitales. La forma más sencilla, pero no más cómoda, es suscribirse a las revistas que contengan artículos de interés o escribirle personalmente al autor y solicitarle una copia de su artículo. ${ }^{16}$

\section{TÉRMINOS MeSH Y DESC}

Realizar búsquedas en estas bases de datos puede resultar complicado si no se usan las palabras y la técnica correcta, es por esto que lo más recomendable es utilizar los Medical Subject Headings, conocidos como términos MeSH en inglés y en español designados como términos DeSC (Descriptores en Ciencia de la Salud). ${ }^{12,17}$ Estos nos permiten delimitar más la búsqueda y evitar encontrar o leer artículos innecesarios. Es decir, que nos hacen más fácil la identificación de literatura relevante, en asociación con el tema de interés. ${ }^{3,17}$ Estos términos se encuentran estandarizados a nivel mundial, y organizados jerárquicamente por conceptos, formando lo que se conoce como el árbol de temas. Esta disposición permite a los usuarios buscar según los niveles de especificidad. ${ }^{3}$ Estos términos no deben ser conocidos de memoria, y de hecho existen buscadores a la disposición de todos para cerciorarse que el término uti- lizado sea el apropiado a nivel científico. Se recomienda que, al escribir un artículo, las palabras clave del mismo sean términos $\mathrm{MeSH}$ o DeSC, por motivos de indexación y facilidad de la búsqueda posterior. ${ }^{18}$

\section{CONCLUSIÓN}

La búsqueda en base de datos es una actividad que resulta cómoda para quien conoce las técnicas, estrategias y herramientas aquí explicadas; y una tarea desgastante para aquellos que no las implementan. Estas bases de datos son una fuente rica de conocimientos a disposición de todos, y es necesario aprender a usarlas para nuestro beneficio, de esta forma se realizarán búsquedas adecuadas que no solo nos permitirán nutrirnos de conocimientos comprobados y correctos, sino que también nos otorga la posibilidad de elaborar trabajos investigativos sólidos y de alto nivel. La correcta utilización de términos MeSH o DeSC permitirá poner a disposición de la comunidad biomédica material con información actualizada que servirá para mejorar la salud de los pacientes.

\section{REFERENCIAS BIBLIOGRÁFICAS}

1. Sindhu F, Dickson R. The complexity of searching the literature. Int J Nurs Pract. diciembre de 1997;3(4):211-7.

2. Gómez Caridad I, Fernández Muñoz MT, Bordons Gangas M, Morillo Ariza F. La producción científica española en Medicina en los años 1994-1999. Rev Clínica Esp. 2004;204(2):75-88.

3. Fine EV, Bliss DZ. Searching the literature: understanding and using structured electronic databases. J Wound Ostomy Cont Nurs Off Publ Wound Ostomy Cont Nurses Soc WOCN. diciembre de 2006;33(6):594-605.

4. Buñuel Âlvarez JC, González Rodríguez MP. Búsquedas bibliográficas a través de Internet. como encontrar la mejor evidencia disponible: Bases de datos de medicina basada en la evidencia. Rev Pediatría Aten Primaria. 2003;5(17):109-32.

5. Ospina EG, Herault LR, Cardona AF. Uso de bases de datos bibliográficas por investigadores biomédicos latinoamericanos hispanoparlantes: estudio transversal. Rev Panam Salud Pública. abril de 2005;17(4):230-6.

6. Wang L, Wang J, Wang M, Li Y, Liang Y, Xu D. Using Internet search engines to obtain medical information: a comparative study. J Med Internet Res. 2012;14(3):e74.

7. Rodríguez Camiño R. Motores de búsqueda sobre salud en Internet. ACIMED. octubre de 2003;11(5):0-0. 
8. Wilczynski NL, Haynes RB, Lavis JN, Ramkissoonsingh R, Arnold-Oatley AE, HSR Hedges team. Optimal search strategies for detecting health services research studies in MEDLINE. CMAJ Can Med Assoc J J Assoc Medicale Can. el 9 de noviembre de 2004;171(10):1179-85.

9. Wilczynski NL, Haynes RB. Developing optimal search strategies for detecting clinically sound prognostic studies in MEDLINE: an analytic survey. BMC Med. el 9 de junio de 2004;2:23.

10. Clough JFM, Hitchcock K, Nelson DL. Using PubMed effectively to access the orthopaedic literature. Instr Course Lect. 2011;60:607-18.

11. Sood A, Ghosh AK. Literature search using PubMed: an essential tool for practicing evidencebased medicine. J Assoc Physicians India. abril de 2006;54:303-8.

12. Richter RR, Austin TM. Using MeSH (Medical Subject Headings) to Enhance PubMed Search Strategies for Evidence-Based Practice in Physical Therapy. Phys Ther. el 1 de enero de 2012;92(1):124-32.

13. Bachmann LM, Estermann P, Kronenberg C, ter Riet G. Identifying diagnostic accuracy studies in EMBASE. J Med Libr Assoc JMLA. julio de 2003;91(3):341-6.
14. Wilczynski NL, Haynes RB, Hedges Team. EMBASE search strategies achieved high sensitivity and specificity for retrieving methodologically sound systematic reviews. J Clin Epidemiol. enero de 2007;60(1):29-33.

15. Wong SSL, Wilczynski NL, Haynes RB. Optimal CINAHL search strategies for identifying therapy studies and review articles. J Nurs Scholarsh Off Publ Sigma Theta Tau Int Honor Soc Nurs Sigma Theta Tau. 2006;38(2):194-9.

16. Wolfe N, Gøtzsche PC, Bero L. Strategies for obtaining unpublished drug trial data: a qualitative interview study. Syst Rev. 2013;2:31.

17. Lowe HJ, Barnett $\mathrm{G}$. UNderstanding and using the medical subject headings (mesh) vocabulary to perform literature searches. JAMA. el 13 de abril de 1994;271(14):1103-8.

18. Huang M, Névéol A, Lu Z. Recommending $\mathrm{MeSH}$ terms for annotating biomedical articles. J Am Med Inform Assoc. el 1 de septiembre de 2011;18(5):660-7. 